\title{
Deep learning-based image analysis methods for brightfield-acquired multiplex immunohistochemistry images
}

Danielle J. Fassler ${ }^{1 \dagger}$, Shahira Abousamra ${ }^{2 \dagger}$, Rajarsi Gupta ${ }^{3}$, Chao Chen ${ }^{3}$, Maozheng Zhao ${ }^{2}$, David Paredes ${ }^{2}$, Syeda Areeha Batool ${ }^{3}$, Beatrice S. Knudsen ${ }^{4}$, Luisa Escobar-Hoyos ${ }^{1,5}$, Kenneth R. Shroyer ${ }^{1}$, Dimitris Samaras ${ }^{2}$, Tahsin Kurc $^{3}$ and Joel Saltz ${ }^{3^{*}}$

\begin{abstract}
Background: Multiplex immunohistochemistry ( $\mathrm{mlHC}$ ) permits the labeling of six or more distinct cell types within a single histologic tissue section. The classification of each cell type requires detection of uniquely colored chromogens localized to cells expressing biomarkers of interest. The most comprehensive and reproducible method to evaluate such slides is to employ digital pathology and image analysis pipelines to whole-slide images (WSIs). Our suite of deep learning tools quantitatively evaluates the expression of six biomarkers in mIHC WSIs. These methods address the current lack of readily available methods to evaluate more than four biomarkers and circumvent the need for specialized instrumentation to spectrally separate different colors. The use case application for our methods is a study that investigates tumor immune interactions in pancreatic ductal adenocarcinoma (PDAC) with a customized $\mathrm{mlHC}$ panel.

Methods: Six different colored chromogens were utilized to label T-cells (CD3, CD4, CD8), B-cells (CD20), macrophages (CD16), and tumor cells (K17) in formalin-fixed paraffin-embedded (FFPE) PDAC tissue sections. We leveraged pathologist annotations to develop complementary deep learning-based methods: (1) ColorAE is a deep autoencoder which segments stained objects based on color; (2) U-Net is a convolutional neural network (CNN) trained to segment cells based on color, texture and shape; and (3) ensemble methods that employ both ColorAE and U-Net, collectively referred to as ColorAE:U-Net. We assessed the performance of our methods using: structural similarity and DICE score to evaluate segmentation results of ColorAE against traditional color deconvolution; F1 score, sensitivity, positive predictive value, and DICE score to evaluate the predictions from ColorAE, U-Net, and ColorAE:U-Net ensemble methods against pathologist-generated ground truth. We then used prediction results for spatial analysis (nearest neighbor).

(Continued on next page)
\end{abstract}

\footnotetext{
* Correspondence: joel.saltz@stonybrookmedicine.edu

${ }^{\dagger}$ Danielle J. Fassler and Shahira Abousamra contributed equally to this work. ${ }^{3}$ Department of Biomedical Informatics, Stony Brook University Renaissance School of Medicine, 101 Nicolls Rd, Stony Brook 11794, USA

Full list of author information is available at the end of the article
}

(c) The Author(s). 2020 Open Access This article is licensed under a Creative Commons Attribution 4.0 International License, which permits use, sharing, adaptation, distribution and reproduction in any medium or format, as long as you give appropriate credit to the original author(s) and the source, provide a link to the Creative Commons licence, and indicate if changes were made. The images or other third party material in this article are included in the article's Creative Commons licence, unless indicated otherwise in a credit line to the material. If material is not included in the article's Creative Commons licence and your intended use is not permitted by statutory regulation or exceeds the permitted use, you will need to obtain permission directly from the copyright holder. To view a copy of this licence, visit http://creativecommons.org/licenses/by/4.0/. The Creative Commons Public Domain Dedication waiver (http://creativecommons.org/publicdomain/zero/1.0/) applies to the data made available in this article, unless otherwise stated in a credit line to the data. 
(Continued from previous page)

Results: We observed that (1) the performance of ColorAE is comparable to traditional color deconvolution for single-stain IHC images (note: traditional color deconvolution cannot be used for mIHC); (2) ColorAE and U-Net are complementary methods that detect six different classes of cells with comparable performance; (3) combinations of ColorAE and U-Net in ensemble methods outperform ColorAE and U-Net alone; and (4) ColorAE:U-Net ensemble methods can be employed for detailed analysis of the tumor microenvironment (TME).

Summary: We developed a suite of scalable deep learning methods to analyze 6 distinctly labeled cell populations in mIHC WSIs. We evaluated our methods and found that they reliably detected and classified cells in the PDAC tumor microenvironment. We also utilized the ColorAE:U-Net ensemble method to analyze 3 mIHC WSIs with nearest neighbor spatial analysis. We demonstrate a proof of concept that these methods can be employed to quantitatively describe the spatial distribution of immune cells within the tumor microenvironment. These complementary deep learning methods are readily deployable for use in clinical research studies.

Keywords: Multiplex immunohistochemistry, Digital pathology image analysis, Deep learning, Tumor immune microenvironment

\section{Background}

Multiplex IHC (mIHC) and multiplex immunofluorescence (mIF) are methods that are used to detect multiple targets in a single histologic section with different colored chromogens (e.g. DAB, AES, TMB, BCIP) or fluorophores for $\mathrm{mIHC}$ and $\mathrm{mIF}$, respectively. Traditional IHC employs a single antibody for each tissue section, where multiple markers are assessed in consecutive serial tissue sections. Therefore, mIHC increases our ability to observe direct interactions between cells within the appropriate histological context in a single tissue section and maximizes the number of markers that can be assessed with limited tissue. Fully automated mIHC and mIF platforms are being deployed as high-throughput assays for future use in CLIA/ CAP certified laboratory settings.

We used a mIHC platform to visualize inflammatory responses in the tumor microenvironment of pancreatic ductal adenocarcinoma (PDAC). We chose this model system since PDAC is one of the deadliest types of cancer, known to be poorly immunogenic and unresponsive to currently available immunotherapeutic treatment options [1, 2]. Investigation of the relationship between PDAC and the inflammatory microenvironment could be further advanced by the development of methods that quantify cell populations and their distribution within the tumor microenvironment in an automated and reproducible fashion. We utilized $\mathrm{mIHC}$ rather than $\mathrm{mIF}$ due to the decay of fluorophores over time, challenges associated with interpreting mIF from the lack of histologic context, and need for specialized fluorescence or spectral imaging instrumentation that is labor intensive, expensive, and requires expertise [3-20].

The analysis of inflammatory responses in the tumor microenvironment (TME) is increasingly significant as the development and deployment of immunotherapeutic protocols continues to increase for many types of cancer $[2,16,18,21-30]$. Investigations of tumor-immune interactions in the TME using $\mathrm{mIHC}$ may help improve clinical outcomes through the discovery of predictive and prognostic biomarkers [14, 26, 27, 30-64]. Since tumor-immune interactions are exquisitely complex and diverse across different types and subtypes of cancer, meaningful analysis of the TME requires the detection and classification tumor cells and immune cell subtypes to (1) characterize the functional immune status of the TME, (2) identify potential intrinsic immune biomarkers, and (3) provide insight into the expression of known immunotherapeutic drug targets. In order to clinically implement mIHC, pathologists have to be able to meaningful interpret multicolored tissue sections that contain several types of labeled cells.

Thus, computational methods are being explored to augment traditional histologic examination in an effort to help reliably detect and classify multiple distinct cell populations in digital whole slide images (WSIs) of mIHC-stained tissue sections $[4,6,7,9,11,13-15,19$, 65-67]. We developed a suite of algorithms that leverage deep learning to overcome the need to use specialized multispectral imaging instrumentation for quantitative analysis of mIHC WSIs containing six or more distinctly colored chromogens. Our methods utilize computationally inexpensive deep learning convolutional neural networks (CNNs) that are trained to separate colors and classify cells in a time efficient and comprehensive manner with limited training data. The success of each of the methods demonstrates the value of using deep learning-based image analysis methods for automated analysis of mIHC WSIs. Therefore, we also present an application of our methods to quantitatively describe the spatial relationships between tumor and immune cells in PDAC as an example of the types of insights that can be gained from such analysis.

We report our efforts to develop and test complementary color deconvolution and immune cell classification 
methods by using deep learning CNNs. We developed a suite of deep learning tools with two distinct algorithmic approaches and combinations of these methods. Our suite of deep learning tools includes (1) a deep autoencoder for color decomposition, (2) a U-Net based approach for cell segmentation, and (3) multiple ensemble approaches intended to increase the positive predictive value (PPV) of cell detection and classification. This manuscript reports the development of these methods in a specific use case to quantitatively analyze the expression of six biomarkers to study tumor immune interactions in PDAC. Our goal was to develop these methods to build robust and scalable analytic pipelines that can be easily configured and deployed to analyze mIHC WSIs for a wide array of research and clinical applications.

\section{Methods}

The identification of different types of cells with mIHC is based on the unique colors of chromogenic agents that are localized to specific cells in formalinfixed and paraffin-embedded (FFPE) tissue sections. However, available research and commercial software used for color decomposition is typically limited to three or four channels. Therefore, we developed multiple deep learning models with training data for six different colored chromogens in a PDAC mIHC panel. Our suite of deep learning tools included: (1) ColorAE, (2) U-Net, and (3) ColorAE:U-Net ensemble models to detect color-labeled immune cell types and tumor cells in mIHC WSIs without the need for spectral deconvolution during image acquisition by digital slide scanners.

\section{Tissue specimens}

FFPE tissue sections ( $5 \mu \mathrm{m}$ thickness) from ten cases of PDAC, provided by the archival collections of the Department of Pathology at Stony Brook University Hospital, were obtained for this mIHC pilot study. These cases represent a subset of cases from a previously published cohort [68]. Tumor sections were reviewed from each case to identify the tissue block with the greatest area of viable tumor and those with $<1 \mathrm{~cm}^{2}$ of tumor were excluded. The tissue slides were de-identified and multiple security measures, including password protection and storage of the password key on a computer without network access were used to ensure that no patient identifiers could be accessed. All of the members of our research team members have undergone CITI human subjects and medical ethics training.

\section{Multiplex IHC}

mIHC of the tissue sections was performed at Roche Diagnostics (Tuscon, AZ), using a Discovery Ultra
Autostainer (Roche/Ventana, Oro Valley, AZ). Tissue slides were baked at 60 degrees Celsius for $20 \mathrm{~min}$, followed by $3 \times 8$-min deparaffinization cycles, antigen retrieval in high $\mathrm{pH}$ buffer (CC1, Roche/Ventana), and treatment to block endogenous peroxidase (Inhibitor $\mathrm{CM}$, Roche/Ventana). Antibodies for CD3, CD4, CD8, $\mathrm{CD} 16$, and CD20 were provided by Roche/Ventana and an antibody to $\mathrm{K} 17$, a biomarker of the most aggressive subtype of PDAC [68] was provided by KDx Diagnostics (Los Gatos, CA). mIHC staining was performed using horseradish peroxidase (HRP)- and alkaline phosphatase (AP)-based protocols with different colored chromogens (e.g. Yellow:CD3 T-cells, Teal:CD4 helper T-cells, Purple:CD8 cytotoxic T-cells, Red:CD20 B-cells, Black:CD16 myeloid cells, and brown (DAB):cancer cells) [68-71]. Secondary monoclonal antibodies to rabbit primary antibodies for CD16, K17, CD8, and $\mathrm{CD} 4$, and $\mathrm{K} 17$ were conjugated to the HQ hapten; monoclonal antibodies to primary rabbit primary antibodies to $\mathrm{CD} 3$ and $\mathrm{CD} 20$ were conjugated to the NP hapten (Fig. 1G). After each round of staining, antibody complexes were removed using CC2 (Roche/Ventana), a pH 6.0 citrate/acetatebased buffer containing 0.3\% SDS, and heating the slide to 93 degrees for $8 \mathrm{~min}$ [72]. Details of the $\mathrm{mIHC}$ protocol are outlined in supplemental Table 1; a complete list of required reagents including washes and buffers is included in supplemental Table 2.

\section{Optimization of IHC protocol}

Before finalizing the $\mathrm{mIHC}$ protocol, we optimized conditions and validated the staining patterns. Controls for individual antibodies: Using two PDAC cases, we stained 6 serial sections with individual antibodies that followed the sections cut for mIHC (Fig. 1A-B). We confirmed that the quality of staining, color intensity, and patterns of IHC staining in each single-stained slide matched the pattern produced with the same antibody in the $\mathrm{mIHC}$ slide. In addition, we ran negative controls that substituted diluent for each of the primary antibodies and secondary antibodies. Heat denaturation controls: Sensitivity of the antigens to repeated denaturation steps was evaluated in adjacent tissue sections prior to application of the primary antibody. Antigens that were sensitive to repeated denaturation were placed earlier in the sequence.

\section{Image capture and preparation}

After mIHC tissue sections were completed, an Olympus VS120 microscope (Olympus, Tokyo, Japan) was used to scan glass slides and generate digital WSIs at 40x magnification with a resolution of $0.175 \mu \mathrm{m}$ per pixel. WSIs were partitioned into patches in order to obtain training data to develop two distinct deep learning models to detect, classify, and segment distinct types of cells in the 


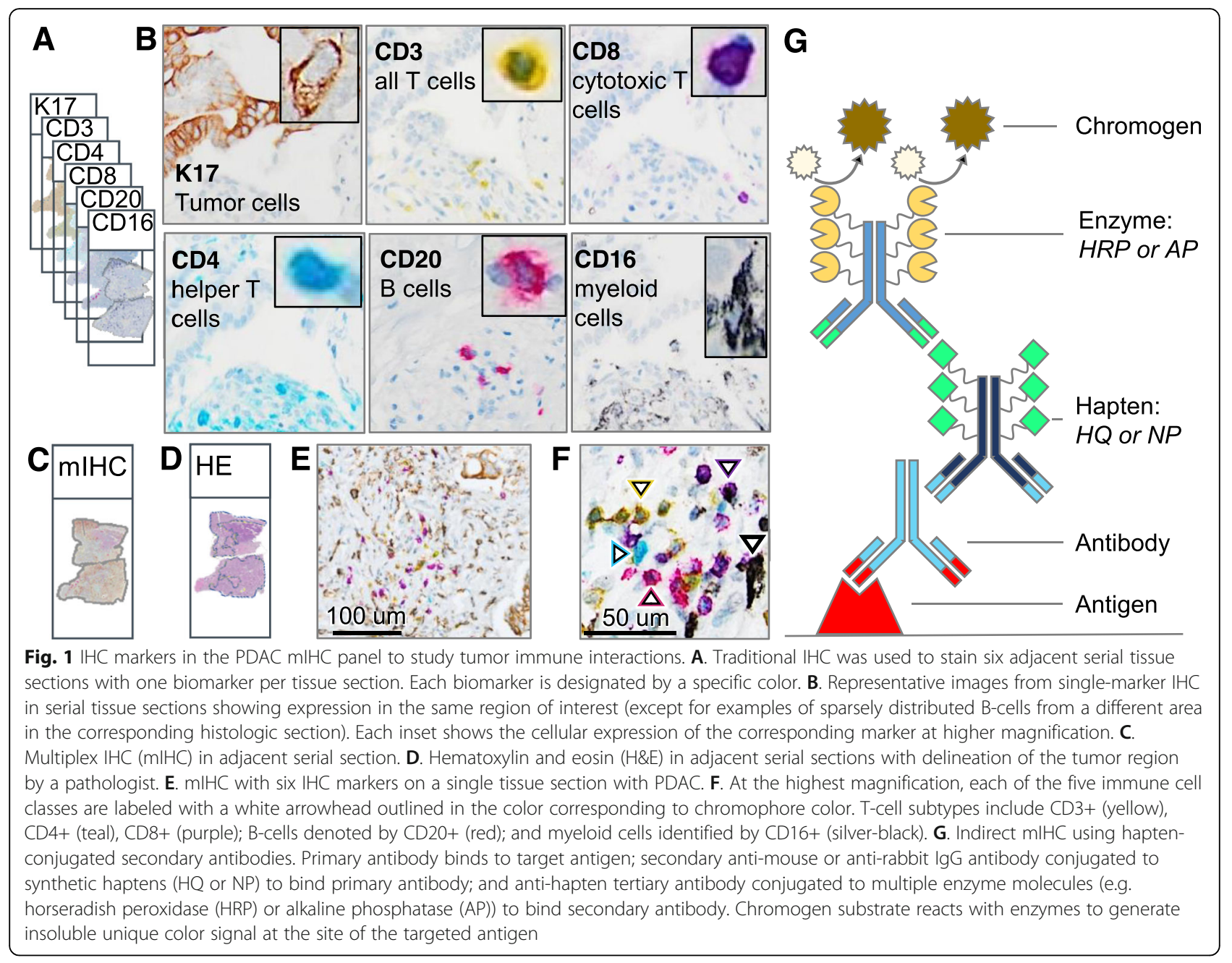

mIHC WSIs. We selected two cases with abundant tissue and obtained six additional serial sections for individually staining with each of the markers in the PDAC mIHC panel for further validation studies.

\section{Generation of ground truth data}

A set of 80 patches $(1920 \times 1200$ pixels $)$ were selected from representative high-density tumor regions from 10 mIHC WSIs. Six cases were used to generate the training dataset (10 patches per case); four separate cases were selected for the test set ( 5 patches per case). Since manually delineating the boundaries of individual cells to provide per-pixel annotations is time and cost prohibitive, we utilized seed labels and superpixels (Fig. 2A, $\mathrm{B}, \mathrm{D})$ to create a relatively large training data set of perpixel annotations (superpixel labels, Fig. 2D). A pathologist examined each patch and placed a seed annotation at the center of each cell to indicate the identity of the cell based on staining. This seed label corresponded to the dominant stain across the cell.
Superpixel computation is a well-developed technique in computer vision [73]. The superpixel method works by partitioning an image into small regions called superpixels, where color is relatively homogeneous within each superpixel (Fig. 2D). Each superpixel containing a seed label is assigned the corresponding label; the remaining superpixels are considered background pixels (Fig. 2D). The resulting superpixel annotations are called super-pixel labels (Fig. 2D). Even though the superpixel label may not exactly match the boundaries of the cells, we were able to improve the strength of the annotations to train the models without increasing the labor needed to generate the labels.

\section{ColorAE}

The color in any given pixel in mIHC WSIs is combination of primary colors. ColorAE predicts the proportion of different colors corresponding to different stains and referred to as color concentration for each pixel (Fig. 3A). By the Beer Lambert Law [74], the summation of the colors of different stains, weighted by their 

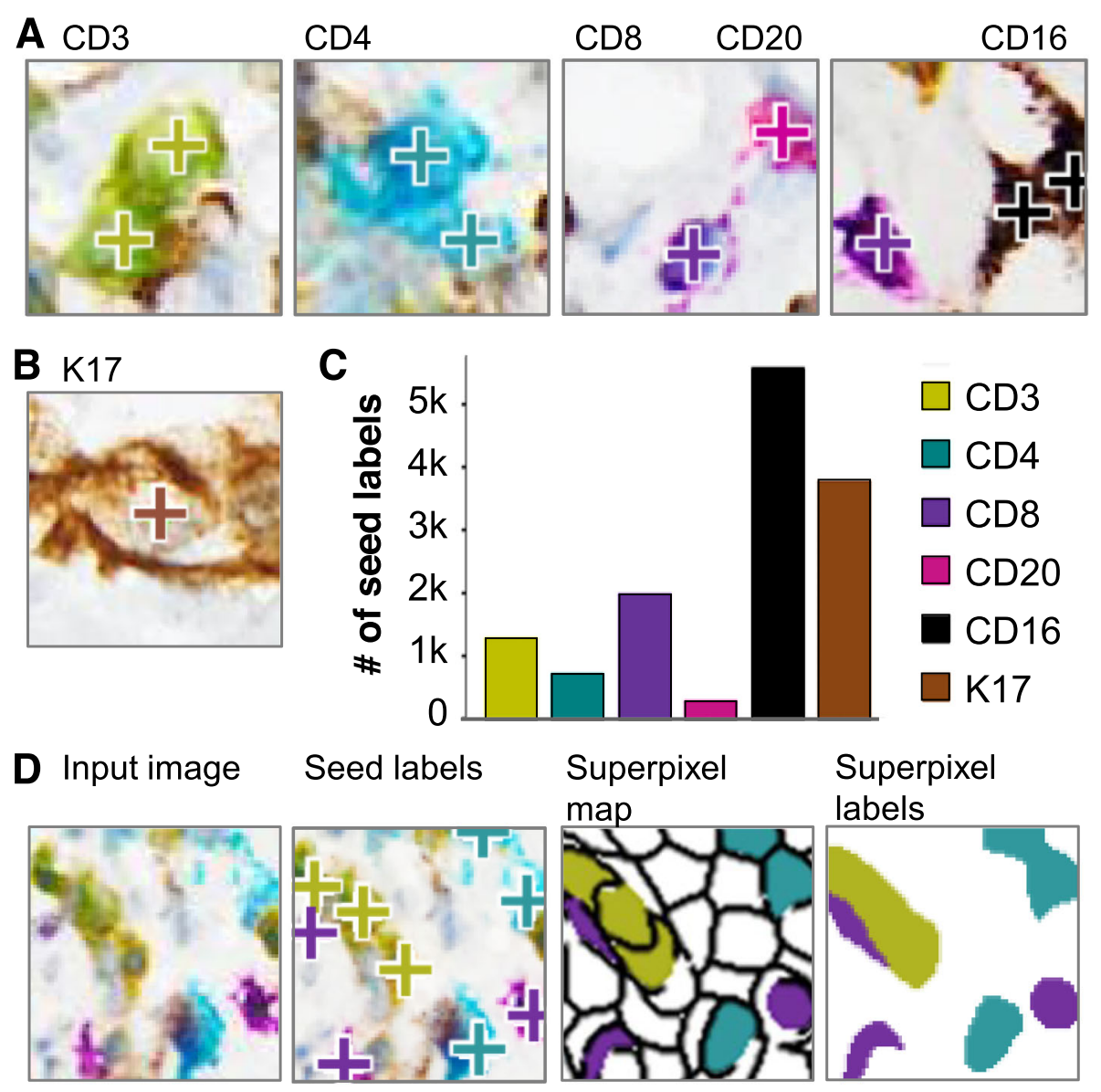

Fig. 2 Annotation of patches with seed labels and generation of per-pixel training data. A. Examples of CD3+, CD4+, CD8+ and CD20+ lymphocytes, CD16+ myeloid cells and B. K17+ PDAC tumor cells with seed labels overlaid (+). C. Number of seed labels for each cell class, across all patches used for training. D. Input image; input image with seed labels overlaid; superpixel map generated based on the input image with superpixels containing different seed labels colored accordingly; and the superpixel labels used to train the models (based on seed labels and superpixel map)

concentrations, is equal to the observed color. This linear relationship is true only after the colors are mapped into optical densities, i.e., the negative logs of the colors after normalization. This provides a means to recover the color concentrations for every pixel when three or fewer colored stains are used by directly solving the linear equation system [75]. If there are more than three stains, the linear equation system becomes underdetermined. Even though one may use more advanced techniques including sparsity regularization and deep neural networks [76-80], these methods do not capture the rich amount of information from colored stains between adjacent pixels, especially for our mIHC images with up to 6 stains.

Our proposed method, ColorAE, is an autoencoder that fully exploits the rich spatial information between stains of adjacent pixels. We briefly introduce the method and published the technical details in parallel (Fig. 3A) [75]. An autoencoder is a deep neural network that applies multiple layers of convolutions to the input image so that it is converted to a low resolution, high dimensional latent space representation (Fig. 3A). A series of deconvolutions are then applied to this latent representation to recover an output of the same resolution of the original image (Fig. 3A). In our case, the neural network is trained to predict an 8-channel image of the same size as the input image. The 8 channels correspond to the concentration maps of six IHC stains, hematoxylin (blue), and background (grey) values (Fig. 3A). More details about the network architecture can be found in [81].

To train the ColorAE model, we introduce two loss functions. A reconstruction loss compares the reconstructed image with the input image pixel-by-pixel by using a mean squared error (MSE) (Fig. 3A). This loss alone is insufficient; due to the excessive number of colored stains, multiple different color decompositions can provide the same reconstruction. In order to find the optimal decomposition solution, we leveraged weak-form supervision from human annotators through a label 


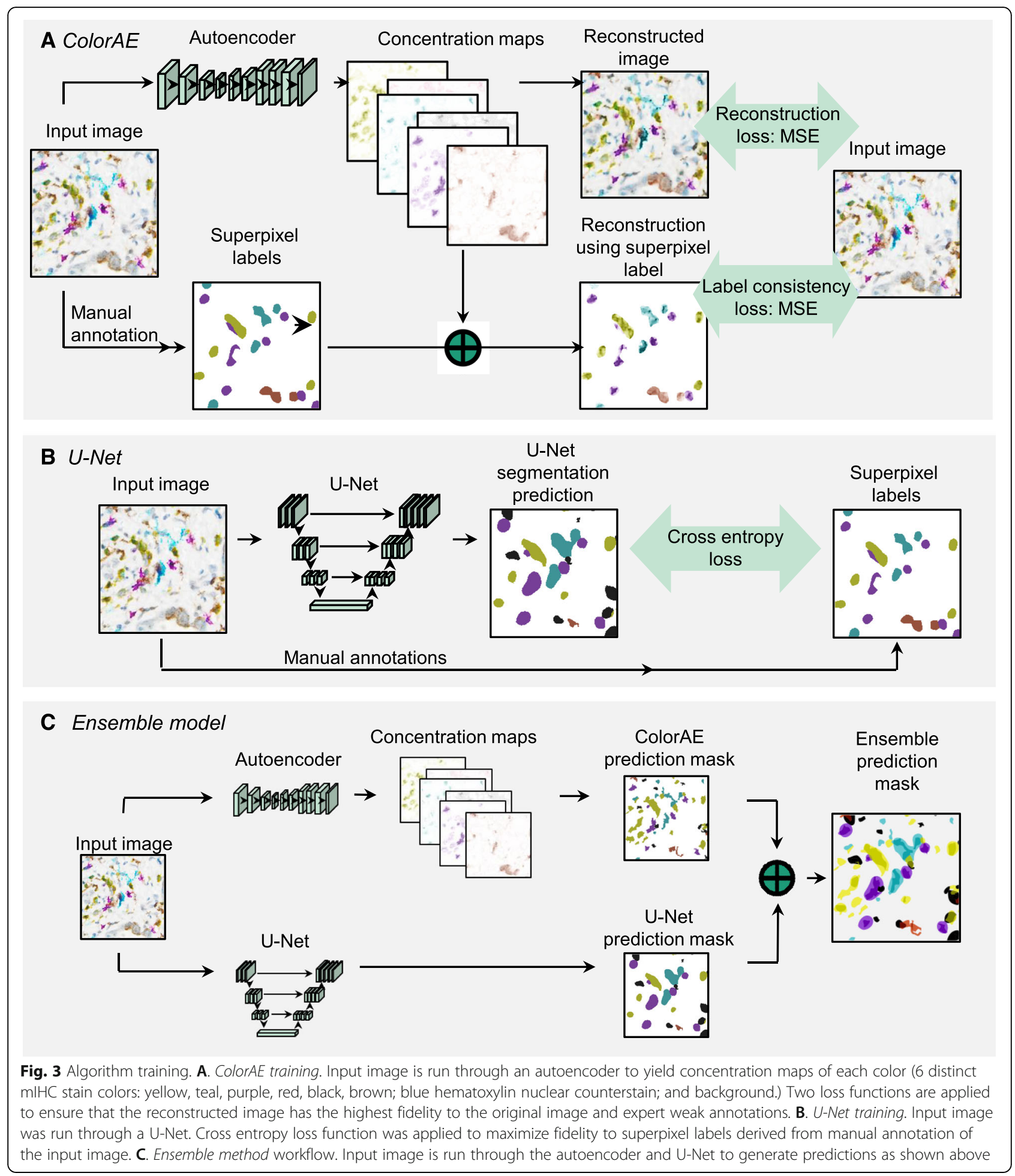

consistency loss function. We created another reconstructed image by using only the superpixel label colored stain and the color concentration value at each pixel (Fig. 3A). By requiring the reconstructed image be as close to the original image, the superpixel label for each pixel is designated as the dominant colored stain.

\section{U-net}

The second method is a segmentation $\mathrm{CNN}$ based on the U-Net architecture [81]. The U-Net architecture is specifically characterized by the skip connections between the encoding and decoding path and has proven efficient in various medical image segmentation tasks 
(Fig. 3B). Similar to ColorAE, this model also uses the superpixel labels. U-Net is trained by minimizing the cross-entropy loss. In particular, the U-Net model is trained with dropout and weighted cross entropy loss in order to account for the class imbalance in the training data and learns to segment the different cell classes. It is different from ColorAE in that it does not try to reconstruct the input image or generate stain concentration maps. U-Net is trained to generate features that differentiate the different cell classes according to the provided labels. In that sense it is less constrained than ColorAE. The resulting segmentation maps are not as fine detailed as ColorAE but prove to provide complementary information as seen visually in Fig. 4 and through evaluation of the ensemble methods.

\section{Ensemble methods}

The ColorAE:U-Net ensemble methods combine the respective strengths of the ColorAE and U-Net deep learning methods (Fig. 3C). Color deconvolution with ColorAE was designed to recover the color composition of an image by predicting the color composition for each pixel, whereas U-Net [81] identifies different types of cells without performing overt color deconvolution. We describe the four ensemble methods below.

Union combines the predictions from both methods; overlapping predictions are combined into a single mask (Fig. 5A,B,D). Intersection only includes pixels identified by both algorithms (Fig. 5E). Union anchor $A E$ takes the union of the masks and discards any U-Net predictions that do not overlap with a ColorAE prediction (only keeps the connected components that contain colored pixels from the ColorAE mask) (Fig. 5F). Union anchor $U$-Net takes the union of the masks and discards any ColorAE predictions that do not overlap with a U-Net prediction (only keeps the connected components that contain colored pixels from the U-Net mask) (Fig. 5G).

\section{Spatial analysis}

We used the results from the best performing algorithm (union anchor AE) for downstream spatial analysis as proof of concept that our algorithm could be employed to survey relationships between cells in the tumor microenvironment. Nearest neighbor distance (NNDist) is determined by the Euclidean length of the shortest vector connecting the edge of one mask or cell (e.g. cytotoxic T-cell) and the edge of the next closest mask or cell (e.g. tumor, as shown in Fig. 6A). In this way, NNDist was calculated with Scipy library to determine the average distance between different types of cells with one and another. Median NNDist distances between immune and tumor cells were found by aggregating them from multiple WSIs and averaging across all of the $2000 \times 2000$ pixels tiles within the pathologist annotated tumor region (Fig. 1C-D). The pathologistannotated tumor region was manually annotated based on visual inspection in an adjacent H\&E tissue section. Proximity analyses utilized NNDist data and counted the number of immune cells within discrete distance intervals from the nearest tumor mask.
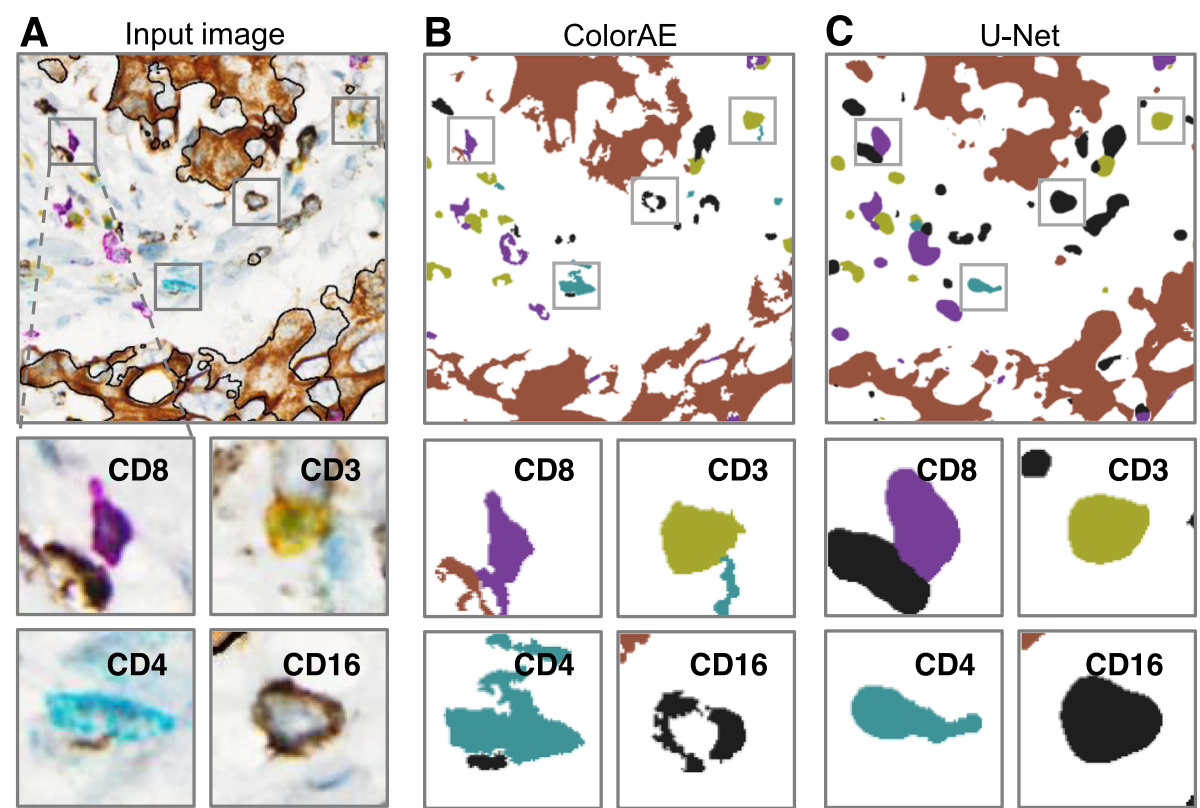

Fig. 4 ColorAE and U-Net predictions. A. mIHC input image of tumor microenvironment, with a representative cell from each class of immune cells (magnified below). B-C. ColorAE and U-Net prediction masks based on the original image 


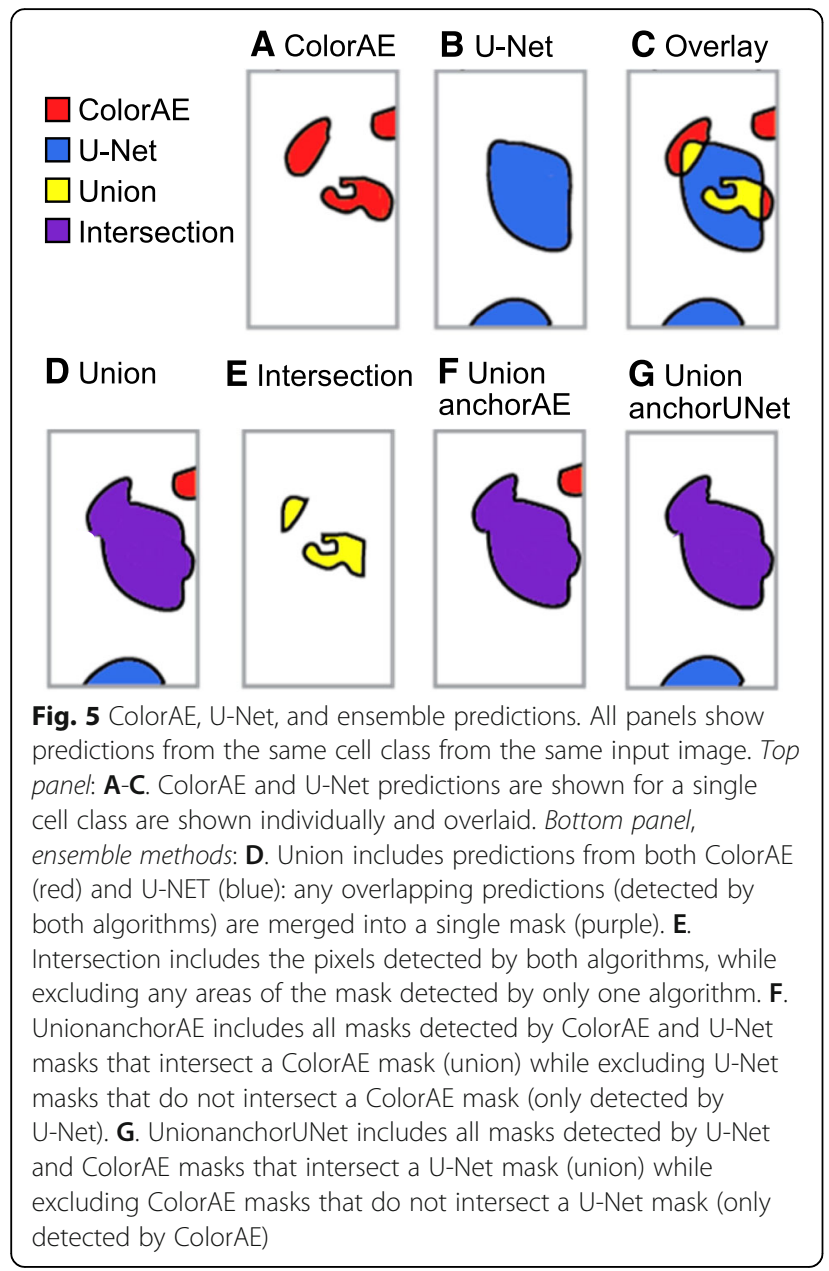

\section{Results}

We evaluated the performance of the proposed methods by using (1) structural similarity and DICE score to compare results from ColorAE to traditional color deconvolution used on a set of consecutive serial tissue sections stained with single colors, (2) F1 score, sensitivity (recall), and positive predictive value (precision) comparing predictions of all algorithms to dilated seed labels, and (3) DICE score to compare predictions of all algorithms with hand-drawn annotations (referred to here as per-pixel labels).

\section{Evaluation of similarity between ColorAE and color deconvolution approaches on single-stain images} The ColorAE method was explicitly designed to detect different colored stains through classical color decomposition for the single-stained images since they consisted of only three colors [80]. We should note that this method was designed for H\&E images and it is limited to the analysis of no more than three colored stains. It cannot be applied to $\mathrm{mIHC}$ images. By visual inspection, the results from ColorAE were comparable to those predicted by Vahadane's method with the notable exception of CD16. This appears to be due to the similarity between black stain and the gray background color and the inability of ColorAE to reliably distinguish between the two in the context of single-stained images (Fig. S1). We utilized the mean structural similarity index (SSim) in 20 randomly selected patches $(1440 \times 1440$ pixels) to quantitatively compare ColorAE and Vahadane's method. SSim [73] measures the similarity between two predictions with respect to luminance, contrast, and structure over sliding windows. SSim is particularly more robust than traditional methods to measure image prediction quality (e.g. peak signal to noise ratio (PSNR) and mean squared error (MSE)). We also compared masks from the derived segmentation of colored stains, which are computed from automatic determination of color concentration, to the masks from Vahadane's method in [79] by using the DICE score (Fig. S1, Table 1). The DICE score is computed as twice the area of intersection of the two masks divided by the sum of their individual areas, formally, $\mathrm{DSC}=2|\mathrm{~A} \cap \mathrm{B}| /$ $(|\mathrm{A}|+|\mathrm{B}|)$, where $\mathrm{A}$ and $\mathrm{B}$ are the regions from the predicted mask and the ground truth mask, respectively.

\section{Evaluation of label prediction performance against dilated seed labels}

The next set of experiments evaluated the performances of ColorAE, U-Net, and the ensemble methods by comparing the models' predictions with the ground truth seed labels (Fig. 5). We dilated the seed labels into disks with a diameter of $10.5 \mathrm{um}$ to correspond to the average size of lymphocytes. The masks for each colored stain were evaluated one at a time. Using the predictions and dilated seed labels, we evaluated true positives (TP), false positives (FP), and false negatives (FN). Specifically, TP is the number of connected components in the mask overlapping with the $10 \mu \mathrm{m}$ disks; FP is the number of connected components in the mask that do not overlap with any disks; and FN is the number of disks that do not overlap with the mask. Since some cell types might be sparse in some patches, we aggregated values for TP, FP, and FN from all of the 20 testing patches. These aggregated values were used to compute standard performance metrics like the F1-score, recall, and precision (Tables 2, 3 and 4).

\section{Comparison of a U-net trained with dilated seed labels to U-net trained with superpixel labels}

We also compared standard U-Net to the superpixel training labels. Generation of sufficient per-pixel annotations for training is prohibitively expensive. As an alternative to superpixel labels, we dilated the seed labels into disks with a small, conservative, diameter of 2 um and use these masks as training data to train a U-Net. We focused on immune cell markers (CD3, CD4, CD8, $\mathrm{CD} 20)$ as lymphocytes are relatively regular in size and 


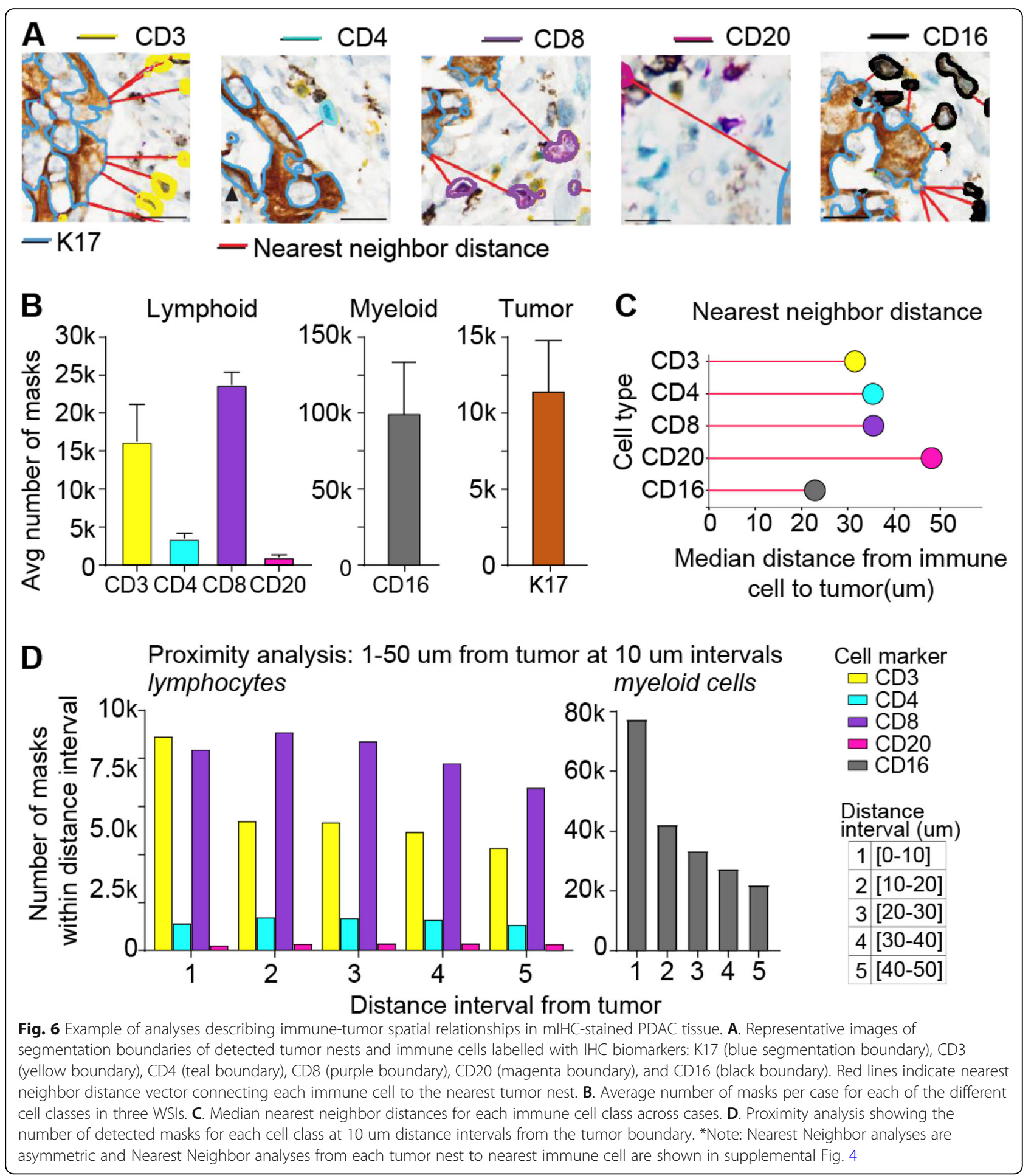

shape and we did not include CD16 and K17 in this evaluation since the myeloid and tumor cells are irregular in size and shape. The results are reported in supplemental material (Fig. S2, Table S3). We show that U-Net trained with superpixel labels significantly outperforms the baseline U-Net trained on dilated disks.
Evaluation of label predictions against hand-drawn perpixel annotations

In the final set of experiments, we generated finegrained hand-drawn per-pixel segmentation annotations (Fig. S3I-J) in a small test set of 19 patches $(470 \times 470$ pixels) and evaluated performance using the DICE score. 
Table 1 Evaluation of ColorAE

\begin{tabular}{lllllll}
\hline Evaluation metrics & CD3 & CD4 & CD8 & CD20 & CD16 & K17 \\
\hline SSim (color concentration. & 0.9 & 0.87 & 0.9 & 0.87 & 0.54 & 0.94 \\
DICE (segmentation masks. & 0.87 & 0.81 & 0.86 & 0.34 & 0.59 & 0.73 \\
\hline
\end{tabular}

The DICE score was used to compare the prediction masks (Fig. S3A-H) with the ground truth mask for each colored stain (Table 5).

\section{Analysis of the PDAC tumor microenvironment}

Union anchor U-Net was used to generate predictions for tumor and immune cells throughout the tumor region of three mIHC WSIs (Fig. 6A). We calculated both the number of masks per cell class and area of each mask. Our analyses show that the immune microenvironment is dominated by CD16+ myeloid cells (Fig. 6B, S4A). Nearest neighbor distances were calculated between each immune cell and the nearest tumor mask, minimizing vector length (Fig. 6A). The average myeloid cell was also closer to tumor cells than any lymphoid cell class was. We observed that on average, CD3 + CD4CD8- T-cells, CD4+ helper T-cells, and CD8+ cytotoxic $\mathrm{T}$-cells were about $11 \mathrm{um}$ further from tumor cells than myeloid cells were, whereas B-cells were 13 um further away than T-cells were (Fig. 6C). The median nearest neighbor distance from tumor to immune cells are 2.9 um for CD16+ myeloid, 23.2 um for CD3 + CD4-CD8T-cells, 44.5 um for CD4+ helper T-cells, 24.0 for CD8+ cytotoxic T-cells, and $56.9 \mu \mathrm{m}$ for CD20+ B-cells, respectively (Fig. S4). (Note: Nearest Neighbor analyses are asymmetric and there is a difference between calculating the distances from "immune cells to tumor" versus "tumor to immune cells." The proximity analysis shows the number of masks (cells of each immune cell class) at increasing distance intervals from tumor cells. Looking at $10 \mu \mathrm{m}$ intervals starting at the tumor mask boundary, the number of myeloid masks drops significantly with increasing distance from the tumor, in contrast to lymphocytes (Fig. 6D). When looking at $1 \mu \mathrm{m}$ intervals from 0 to $5 \mu \mathrm{m}$ from the tumor mask boundary, we see that about 30,000 nearest neighbor myeloid cells were within

Table 2 F1 Scores evaluating predictions from UNet, ColorAE, and ensemble methods against dilated seed labels

\begin{tabular}{lllllll}
\hline Model & CD3 & CD4 & CD8 & CD20 & CD16 & K17 \\
\hline ColorAE & 0.652 & 0.728 & 0.72 & 0.347 & 0.796 & 0.637 \\
UNet & 0.628 & 0.661 & 0.628 & 0.353 & 0.687 & 0.739 \\
Intersection & $\mathbf{0 . 6 9 8}$ & 0.693 & 0.722 & 0.279 & 0.79 & 0.749 \\
Union & 0.593 & 0.705 & 0.628 & $\mathbf{0 . 4 0 3}$ & 0.691 & 0.638 \\
Union anchor AE & 0.662 & $\mathbf{0 . 7 3 2}$ & $\mathbf{0 . 7 3 1}$ & 0.346 & $\mathbf{0 . 8 3 6}$ & 0.657 \\
Union anchor UNet & 0.639 & 0.674 & 0.633 & 0.35 & 0.7 & $\mathbf{0 . 8 0 6}$ \\
\hline
\end{tabular}

Table 3 Sensitivity (Recall) evaluating predictions from UNet, ColorAE, and ensemble methods against dilated eed labels

\begin{tabular}{lllllll}
\hline Model & CD3 & CD4 & CD8 & CD20 & CD16 & K17 \\
\hline ColorAE & 0.974 & 0.798 & 0.868 & 0.212 & 0.914 & 0.871 \\
UNet & 0.931 & 0.675 & 0.881 & 0.232 & 0.991 & 0.756 \\
Intersection & 0.919 & 0.627 & 0.865 & 0.163 & 0.909 & 0.727 \\
Union & $\mathbf{0 . 9 8 1}$ & $\mathbf{0 . 8 4 8}$ & $\mathbf{0 . 8 8 7}$ & $\mathbf{0 . 2 7 5}$ & $\mathbf{0 . 9 9 3}$ & $\mathbf{0 . 9 0 9}$ \\
Union anchor AE & 0.974 & 0.795 & 0.872 & 0.212 & 0.936 & 0.887 \\
Union anchor UNet & 0.933 & 0.686 & 0.884 & 0.229 & 0.992 & 0.846 \\
\hline
\end{tabular}

$1 \mu \mathrm{m}$ (touching) tumor cells, which dropped to approximately 5000 cells and remained steady for the next 4 intervals (Fig. S4). In comparison, $4400 \mathrm{CD} 3+$ CD4-CD8- T-cells, 165 CD4+ helper T-cells, and 1600 CD8+ cytotoxic T-cells were touching tumor (Fig. S4).

\section{Discussion}

The methods described for image analysis of mIHCstained slides were designed to be robust, reliable, and easily customizable for future clinical research applications. We developed our suite of analytic methods in an effort to make a clear and significant advancement in the ability to survey the immune landscape of PDAC using deep learning to help unravel the complexity of tumor immune interactions in the TME. Our goals were to develop a scalable suite of methods to analyze PDAC mIHC WSIs in a uniform manner, where we can (1) reliably detect, classify, and enumerate different cell types labeled with different colored biomarkers, (2) calculate the distances between the boundaries of tumor and immune cells in mIHC WSIs, and (3) perform spatial analyses to quantitatively describe a large number of diverse tumor immune interactions in multicolored $\mathrm{mIHC}$ WSIs without needing expensive multispectral imaging instrumentation.

Our models leverage CNNs trained with this ground truth data to perform pattern recognition functions with statistical multivariate algorithms to predict color and classify all of the different types of labeled cells in the

Table 4 Positive Predictive Value (Precision) evaluating predictions from UNet, ColorAE, and ensemble methods against dilated seed labels

\begin{tabular}{lllllll}
\hline Model & CD3 & CD4 & CD8 & CD20 & CD16 & K17 \\
\hline ColorAE & 0.49 & 0.67 & 0.615 & 0.945 & 0.706 & 0.502 \\
UNet & 0.473 & 0.647 & 0.488 & 0.736 & 0.526 & 0.722 \\
Intersection & $\mathbf{0 . 5 6 2}$ & $\mathbf{0 . 7 7 3}$ & 0.619 & $\mathbf{0 . 9 5 7}$ & 0.699 & $\mathbf{0 . 7 7 1}$ \\
Union & 0.425 & 0.603 & 0.486 & 0.757 & 0.53 & 0.491 \\
Union anchor AE & 0.501 & 0.678 & $\mathbf{0 . 6 2 9}$ & 0.944 & $\mathbf{0 . 7 5 6}$ & 0.521 \\
Union anchor UNet & 0.486 & 0.663 & 0.493 & 0.738 & 0.541 & 0.77 \\
\hline
\end{tabular}


Table 5 DICE Scores evaluating predictions from UNet, ColorAE, and ensemble methods against per-pixel (hand-drawn) labels

\begin{tabular}{lllllll}
\hline Model & CD3 & CD4 & CD8 & CD20 & CD16 & K17 \\
\hline ColorAE & $\mathbf{0 . 6 4 9}$ & 0.543 & 0.764 & 0.589 & 0.539 & $\mathbf{0 . 6 4 9}$ \\
UNet & 0.649 & 0.463 & 0.676 & 0.554 & 0.581 & 0.649 \\
Intersection & 0.457 & 0.457 & $\mathbf{0 . 7 6 9}$ & 0.539 & 0.530 & 0.457 \\
Union & 0.553 & 0.540 & 0.675 & $\mathbf{0 . 5 9 9}$ & 0.591 & 0.553 \\
Union anchor AE & 0.611 & $\mathbf{0 . 5 4 8}$ & 0.685 & 0.588 & $\mathbf{0 . 5 9 3}$ & 0.611 \\
Union anchor UNet & 0.526 & 0.509 & 0.676 & 0.558 & 0.592 & 0.526 \\
\hline
\end{tabular}

PDAC mIHC WSIs. The methods described leverage relatively inexpensive seed labels (dots) that can be used to generate training sets. Importantly, the ability to use this form of annotation significantly decreases the effort for pathologists to generate training data since placing seed labels at the center of each cell is kuch quicker than manually segmenting all of the different types of cells by hand. Significantly reduced time, labor, and cost leads to the ability to quickly customize analytic pipelines and improves the scalability of our methods.

After training, our models, which are sophisticated statistical algorithms, iteratively improve by learning additional features in successive cycles. These deep learning models perform non-linear regression in large data sets to make predictions that can be used to quantitatively analyze the features of the uniquely colored cell types in mIHC WSIs. However, evaluating these algorithms in terms of their ability to correctly identify and classify six distinct cell populations with variable spatial distributions simultaneously in mIHC WSIs requires many considerations.

The variability of shapes and sizes of cells along with the variable expression of each of the biomarkers in individual cells within the different labeled cell population leads to formidable challenges for any pathologist and algorithm. Furthermore, subtle differences in staining patterns coupled with overlapping color spectra of the chromogens introduces difficulty in color decomposition from the very beginning. For example, intense yellow and light black can both appear brown. This is further complicated in cases where a cell class may be labeled with more than one biomarker, e.g., localization of yellow and purple within the same cell can appear red. Thus, we need digital pathology and image analysis tools that can accurately distinguish different cell classes based on the variability of color that depends on how each types of cell is labeled with a particular biomarker in WSIs of mIHC tissue sections. Despite the technical challenges, the proposed ColorAE method generates color decomposition results that are generally consistent with Vahadane's method (as shown in supplemental Fig. 1).
However, ColorAE was designed to analyze mIHC WSIs images with more than three colors. ColorAE performed generally better than U-Net at correctly detecting and classifying multicolored immune cells since ColorAE was able to detect lighter colored immune cells that U-Net failed to detect. We also observed that ColorAE captured fine geometric details that U-Net could not, which is particularly evident when comparing CD8 purple masks. There were also very few B-cells in the tissue sections, which resulted in sample bias, where CD20 red B-cells were often misclassified as CD8 purple Tcells and reflected by the low F1-score. CD16 black myeloid cells and K17 brown PDAC cells were also sometimes difficult to distinguish. Both ColorAE and UNet sometimes misclassified CD16 black as K17 brown and vice versa. Importantly, this seemed to be related in part to the choice of chromogen, where the combination of the black chromogen coupled with the diffuse staining pattern in subsets of myeloid cells appeared brown to the human eye, which can only be distinguished from K17 brown PDAC cells with morphology.

U-Net outperformed ColorAE to detect and classify K17 brown PDAC cells that were counterstained with hematoxylin. Both U-Net and ColorAE can fail to include cell nuclei in the mask since the algorithms generally classify hematoxylin as part of the background. The nuclei of PDAC cells are large and euchromatic with cytoplasmic K17 staining, so it is likely that the algorithms cannot distinguish the nuclei of tumor cells from the background in this use case. Overall, U-Net generally performs better than ColorAE to identify tumor cells. It is important to note that while the tumor cells (and the total tumor mask area) may be underestimated from the exclusion of some nuclei, the boundaries of tumor nests were preserved. Thus, there was still reliable data on tumor nest locations that could be reliably used for downstream spatial analyses.

Furthermore, we show that the methods are complementary, where U-Net had worse recall than ColorAE to detect tumor, but demonstrated significantly better precision. We also observed that ColorAE predicted very detailed masks but was too sensitive in terms of picking up non-specific and background staining. This can be addressed with post-processing by filtering out predictions that contain objects with areas that are below the threshold of being able to be considered as cells. In comparison, the U-Net model produced reasonably conservative predictions, predicting areas of the cell with high intensity staining. However, cells with irregular extensions and low staining intensity were sometimes not detected (Fig. 4). Overall, U-Net performance was limited by the quantity of superpixel labels for training.

In order to address these issues and limitations, we developed the suite of four ColorAE:U-Net ensemble 
models to detect intersections, where a given pixel is predicted to contain a specific color if the pixel is within both of the ColorAE and U-Net masks, and unions, where a pixel is predicted to contain a specific color if the pixel is within either the ColorAE or U-Net masks. We recognize that if each cell class is considered independently, the same pixel may be classified as one class by ColorAE and a different class by U-Net (Fig. 4), so we consider both labels in these scenarios. While sometimes this may be a false positive, in other cases this may be reflective of expression of multiple markers on a single cell (e.g. CD3 + CD4+ cells) that results in compound colors. By treating both of the prediction labels assigned to a given pixel as valid, we can capture this phenomenon to some extent.

Even though the qualitative results from all ColorAE, U-Net, and the ensemble methods are generally acceptable, the Union ensemble demonstrated the best sensitivity (recall), as shown in Table 3 . This is to be expected as the Union ensemble considers pixels positive for each color if the colored label is predicted by at least one model. In terms of precision, the Intersection ensemble demonstrated the best overall positive predictive value (precision) as shown in Table 4, whereas the Union anchor $\mathrm{AE}$ demonstrated the best overall F1 score is considered as shown in Table 2, even though the F1 scores are not directly applicable as a performance metric due to intrinsic variability in the intensity and staining patterns of biomarkers in cells. Although we report considerable progress in developing methods that measure six or more different colored biomarkers in mIHC WSIs, we have to note that these models were trained with a limited dataset and were trained to achieve reasonably good overall performance.

Our results indicate that (1) there is no single universal method that can be the best across all of the performance metrics to target every one of the colored IHC markers and (2) multiple complementary methods can be utilized in analytic pipelines to improve the overall reliability of using computational analysis for mIHC WSIs. In our current use case, we used these novel methods to evaluate the tumor microenvironment PDAC mIHC WSIs. While our focus was to create and evaluate methods for the accurate automated detection of the immune cells in mIHC WSIs, we wanted to demonstrate the types of downstream analyses that can be done to investigate spatial relationships between cell subsets. The nearest neighbor and proximity analyses are based on the spatial positions of all masks across the entirety of the tumor region from a representative PDAC mIHC WSI. For the sake of providing a concrete example, we demonstrate proof that our methods can be used to comprehensively analyze collections of mIHC WSIs.
We emphasize that these methods are still experimental, being refined, and require further comprehensive testing and validation in additional mIHC studies. For example, we observed that segmentation of the boundaries of large PDAC tumor nuclei were occasionally suboptimal and sometimes not detected based on tumor morphology, overlapping nuclei, and obscured nuclear boundaries from intense staining. Even though this limitation can potentially pose a problem with respect to accurately counting every tumor cell, it may not be a significant issue in terms of downstream analyses, including nearest neighbor spatial analyses, since the overall edges of the tumor nests are accurate enough to determine the center point and perimeters of the masks. Nonetheless, the area of K17 brown staining or the number of pixels belonging to $\mathrm{K} 17$ masks can still be calculated in order to provide a reasonable estimate of tumor area.

During the microscopic examination of multicolored PDAC mIHC WSIs, what one commonly observes is a fascinating distribution of classical DAB brown-stained $\mathrm{K} 17+$ PDAC cells in close proximity to an abundance of black-silver colored CD16+ myeloid cells (e.g. macrophages) with variably interspersed purple colored CD3 + CD8+ T-cells, teal colored CD3 + CD4+ T-cells, and yellow CD3 + CD4-CD8- T-cells. We also have observed that red colored $\mathrm{CD} 20+\mathrm{B}$-cells are usually rare in the immune infiltrate associated with PDAC tumor cells, but present in lymphoid aggregates much further away. After histologic review, we utilized our suite of methods to perform spatial analyses in an effort to evaluate the feasibility of quantitatively describing the immune landscape in our PDAC mIHC study. The spatial analyses show how the TME of these PDACs is rich in myeloid cells with a relative dearth of $\mathrm{T}$-cells and B-cells. We also gained insight into patterns of distribution of the three different populations of T-cells. Interestingly, we observed that a significant proportion of the yellow CD3 + CD4-CD8- T-cells may actually represent NK/T-cells, gamma-delta T-cells, or immature T-cells, which can be used to guide other studies.

We are eager to explore whether increasing the size of the cohort will allow us to determine if these patterns are conserved across different cases of PDAC. Furthermore, we are examining the relationship of the spatial patterns of distributions of their different immune cell types with survival data to identify potential prognostic biomarkers. We are also engaged in ongoing studies that are applying these deep learning analytic methods across a much larger cohort of PDAC mIHC WSIs. Future work will also evaluate the relationships between different types of immune cells beyond tumor immune interactions in an effort to better understand cancer immunology. 


\section{Conclusions}

We developed a suite of deep learning tools that can be used to create customizable analytic pipelines to analyze mIHC WSIs with good overall performance in a scalable manner. This suite of tools can be reliably implemented to perform cell detection and classification to explore near limitless combinations of multiple IHC biomarkers in one tissue section. To provide a proof of concept, we presented a novel technique to utilize these computational deep learning analyses to enumerate and characterize the spatial distributions of different types of cells in the TME of selected PDAC mIHC WSIs. Since advances in immunotherapy will likely coincide with increased clinical interest in the functional immune status of TME, we believe that these novel deep learning methods complement the adoption of digital pathology and the potential to deploy mIHC for diagnostic testing of cancer tissue specimens. Thus, we hope that these methods facilitate more widespread adoption of mIHC to support precision medicine and accelerate the discovery of biomarkers that can help predict prognosis and guide treatment.

\section{Supplementary information}

Supplementary information accompanies this paper at https://doi.org/10. 1186/s13000-020-01003-0.

Additional file 1: Supplemental material.

\section{Acknowledgements}

We would like to acknowledge Ventana Medical Systems for optimizing the multiplex immunohistochemistry protocol and performing the staining and quality control for this study; Stony Brook Cancer Center Biorepository for expert assistance to obtain tissue specimens (Dr. R. Kew, PhD); and The Research Foundation for The State University of New York at Stony Brook. This work used the Bridges system, which is supported by NSF award number OAC-1445606, at the Pittsburgh Supercomputing Center (PSC). Thanks to PSC's Facilities Technology Group for their contributions to configuring Bridges-Al.

Patent. Shroyer, K, Escobar-Hoyos, L.F. Keratin 17 as a Prognostic and Predictive Marker of Pancreatic Cancer. Stony Brook University Research Foundation. Filed 10/29/15. Pending Provisional Patent Application.

\section{Data availability}

The datasets used and/or analyzed during the current study are available from the corresponding author on reasonable request.

\section{Authors' contributions}

Study concept and design: JS, KRS, LEH, TK, DJF, RG, CC. Development of methodology: JS, TK, DS, RG, DJF, MZ, SA, DP. Acquisition of data: DJF, RG, $S A, M Z, D P, S A B$. Analysis and interpretation of data: JS, KRS, DF, RG, SA, MZ, CC, BK. Writing, editing, review, and intellectual content of the manuscript: JS, DF, RG, SA, CC, TK, KRS, DPM, BK, MZ, LEH, DS, SAB. Study supervision: JS, TK, DS, KRS, RG, CC, DF. Corresponding author: Dr. Joel Saltz, MD, PhD. joel. saltz@stonybrookmedicine.edu. All author(s) read and approved the final manuscript.

\section{Funding}

This work was supported in part by NCI U24CA180924, U24CA18094S1, U24CA215109 and UG3CA225021 and 18-65-SHRO from a Pancreatic Cancer Action Network Translational Research Grant.

\section{Ethics approval and consent to participate}

This study and protocols were approved by the institutional IRB at Stony Brook University.

\section{Consent for publication}

All authors have read and approved the final manuscript.

\section{Competing interests}

The authors declare that they have no competing interests.

\section{Author details}

${ }^{1}$ Department of Pathology, Stony Brook University Renaissance School of Medicine, 101 Nicolls Rd, Stony Brook 11794, USA. ${ }^{2}$ Department of Computer Science, Stony Brook University, 100 Nicolls Rd, Stony Brook 11794, USA.

${ }^{3}$ Department of Biomedical Informatics, Stony Brook University Renaissance School of Medicine, 101 Nicolls Rd, Stony Brook 11794, USA. ${ }^{4}$ Department of Pathology, University of Utah, 2000 Circle of Hope, Salt Lake City, UT 84112, USA. ${ }^{5}$ Department Therapeutic Radiology, Yale University, 15 York Street, New Haven, CT 06513, USA.

Received: 16 November 2019 Accepted: 12 July 2020

Published online: 28 July 2020

References

1. Sahin $1 \mathrm{H}$, et al. Immunotherapy in pancreatic ductal adenocarcinoma: an emerging entity? Ann Oncol. 2017:28(12):2950-61.

2. Blando J, et al. Comparison of immune infiltrates in melanoma and pancreatic cancer highlights VISTA as a potential target in pancreatic cancer. Proc Natl Acad Sci U S A. 2019;116(5):1692-7.

3. Burugu S, Asleh-Aburaya K, Nielsen TO. Immune infiltrates in the breast cancer microenvironment: detection, characterization and clinical implication. Breast Cancer. 2017;24(1):3-15.

4. Lee SS, et al. Nondestructive, multiplex three-dimensional mapping of immune infiltrates in core needle biopsy. Lab Investig. 2019;99(9):1400-13.

5. $\mathrm{Ma} Z$, et al. Data integration from pathology slides for quantitative imaging of multiple cell types within the tumor immune cell infiltrate. Diagn Pathol. 2017;12(1):69

6. Barua S, et al. Spatial interaction of tumor cells and regulatory T cells correlates with survival in non-small cell lung cancer. Lung Cancer. 2018; 117:73-9.

7. Blom S, et al. Systems pathology by multiplexed immunohistochemistry and whole-slide digital image analysis. Sci Rep. 2017;7(1):15580.

8. Gibney GT, Weiner LM, Atkins MB. Predictive biomarkers for checkpoint inhibitor-based immunotherapy. Lancet Oncol. 2016;17(12):e542-51.

9. Gorris MAJ, et al. Eight-color multiplex immunohistochemistry for simultaneous detection of multiple immune checkpoint molecules within the tumor microenvironment. J Immunol. 2018;200(1):347-54.

10. Halse $\mathrm{H}$, et al. Multiplex immunohistochemistry accurately defines the immune context of metastatic melanoma. Sci Rep. 2018;8(1):11158.

11. Hofman P, et al. Multiplexed immunohistochemistry for molecular and immune profiling in lung Cancer-just about ready for prime-time? Cancers. 2019:11(3):283.

12. Huang W, Hennrick K, Drew S. A colorful future of quantitative pathology: validation of Vectra technology using chromogenic multiplexed immunohistochemistry and prostate tissue microarrays. Hum Pathol. 2013; 44(1):29-38

13. Ilie $\mathrm{M}$, et al. Automated chromogenic multiplexed immunohistochemistry assay for diagnosis and predictive biomarker testing in non-small cell lung cancer. Lung Cancer. 2018;124:90-4.

14. Kalra J, Baker J. Multiplex Immunohistochemistry for Mapping the Tumor Microenvironment. In: Kalyuzhny AE, editor. Signal Transduction Immunohistochemistry: Methods and Protocols. New York: Springer New York; 2017. p. 237-51.

15. Koh J, et al. High-throughput multiplex Immunohistochemical imaging of the tumor and its microenvironment. J Korean Cancer Assoc. 2019;0(0):0-0.

16. Parra ER, Francisco-Cruz A, Wistuba II. State-of-the-Art of Profiling Immune Contexture in the Era of Multiplexed Staining and Digital Analysis to Study Paraffin Tumor Tissues. Cancers (Basel). 2019;11(2).

17. Remark R, et al. In-depth tissue profiling using multiplexed immunohistochemical consecutive staining on single slide. Sci Immunol. 2016;1(1):aaf6925. 
18. Salgado $R$, et al. The evaluation of tumor-infiltrating lymphocytes (TILs) in breast cancer: recommendations by an international TILs working group 2014. Ann Oncol. 2015;26(2):259-71.

19. Stack EC, et al. Multiplexed immunohistochemistry, imaging, and quantitation: a review, with an assessment of Tyramide signal amplification, multispectral imaging and multiplex analysis. Methods. 2014;70(1):46-58.

20. Tsujikawa T, et al. Quantitative multiplex immunohistochemistry reveals myeloid-inflamed tumor-immune complexity associated with poor prognosis. Cell Rep. 2017;19(1):203-17.

21. Palucka AK, Coussens LM. The basis of Oncoimmunology. Cell. 2016;164(6): 1233-47.

22. Parish CR. Cancer immunotherapy: the past, the present and the future. Immunol Cell Biol. 2003;81(2):106-13.

23. Patel SP, Kurzrock R. PD-L1 expression as a predictive biomarker in Cancer immunotherapy. Mol Cancer Ther. 2015;14(4):847-56

24. Roma-Rodrigues $C$, et al. Targeting tumor microenvironment for Cancer therapy. Int J Mol Sci. 2019;20(4):840.

25. Seager RJ, et al. Dynamic interplay between tumour, stroma and immune system can drive or prevent tumour progression. Convergent Sci Phys Oncol. 2017;3:034002.

26. Sharma P, Allison JP. Immune checkpoint targeting in cancer therapy: toward combination strategies with curative potential. Cell. 2015;161(2):205-14.

27. Sharma $P$, et al. Novel cancer immunotherapy agents with survival benefit: recent successes and next steps. Nat Rev Cancer. 2011;11(11):805-12.

28. Smyth MJ, Dunn GP, Schreiber RD. Cancer immunosurveillance and immunoediting: the roles of immunity in suppressing tumor development and shaping tumor immunogenicity. Adv Immunol. 2006:90:1-50.

29. Smyth MJ, et al. Combination cancer immunotherapies tailored to the tumour microenvironment. Nat Rev Clin Oncol. 2016:13(3):143-58.

30. Thorsson V, et al. The Immune Landscape of Cancer. Immunity. 2018;48(4): 812-+.

31. Amgad, M., et al., Structured crowdsourcing enables convolutional segmentation of histology images. 2019.

32. Amgad $\mathrm{M}$, et al. Joint region and nucleus segmentation for characterization of tumor infiltrating lymphocytes in breast cancer. In: Medical Imaging 2019: Digital Pathology; 2019. International Society for Optics and Photonics.

33. Cooper $L$, et al. Feature-based registration of histopathology images with different stains: an application for computerized follicular lymphoma prognosis. Comput Methods Prog Biomed. 2009:96(3):182-92.

34. Cooper LA, et al. PanCancer insights from the Cancer genome atlas: the pathologist's perspective. J Pathol. 2018;244(5):512-24.

35. Cooper LA, et al. The tumor microenvironment strongly impacts master transcriptional regulators and gene expression class of glioblastoma. Am J Pathol. 2012;180(5):2108-19.

36. Cooper LA, et al. Integrated morphologic analysis for the identification and characterization of disease subtypes. J Am Med Inform Assoc. 2012;19(2): 317-23.

37. Cooper LA, et al. Proc IEEE Int Symp Biomed Imaging; 2011. p. 1624-7.

38. Gajewski TF, Schreiber H, Fu YX. Innate and adaptive immune cells in the tumor microenvironment. Nat Immunol. 2013;14(10):1014-22

39. Gurcan MN, et al. Histopathological image analysis: A review, vol. 2; 2009. p. 147.

40. Gurcan MN, et al. Developing the quantitative histopathology image ontology $(\mathrm{QHIO})$ : a case study using the hot spot detection problem. Biomed Inform. 2017;66:129-35

41. Irshad $\mathrm{H}$, et al. Methods for nuclei detection, segmentation, and classification in digital histopathology: a review-current status and future potential. IEEE Rev Biomed Eng. 2014;7.

42. Janowczyk A, et al. High-throughput biomarker segmentation on ovarian cancer tissue microarrays via hierarchical normalized cuts. IEEE Trans Biomed Eng. 2012;59(5):1240-52.

43. Janowczyk A, et al. A resolution adaptive deep hierarchical (RADHicaL) learning scheme applied to nuclear segmentation of digital pathology images. Comput Methods Biomech Biomed Eng Imaging Vis. 2018:6(3):270-6.

44. Janowczyk A, A.J.J.o.p.i. Madabhushi. Deep learning for digital pathology image analysis: A comprehensive tutorial with selected use cases; 2016. p. 7

45. Kothari $\mathrm{S}$, et al. Pathology imaging informatics for quantitative analysis of whole-slide images. J Am Med Inform Assoc. 2013;20(6):1099-108.
46. Kumar A, et al. Automated analysis of immunohistochemistry images identifies candidate location biomarkers for cancers. Proc Natl Acad Sci. 2014;111(51):18249-54

47. Kumar H, Kawai T, Akira S. Pathogen recognition by the innate immune system. Int Rev Immunol. 2011;30(1):16-34.

48. Madabhushi A, et al. Computer-aided prognosis: predicting patient and disease outcome via quantitative fusion of multi-scale, multi-modal data. Comput Med Imaging Graph. 2011;35(7-8):506-14.

49. Madabhushi A, Lee $\mathrm{G}$. Image analysis and machine learning in digital pathology: challenges and opportunities. Med Image Anal. 2016;33:170-5.

50. Netea $M G$, et al. Trained immunity: A program of innate immune memory in health and disease. Science. 2016;352(6284):aaf1098.

51. Nordstrom RJ. The quantitative imaging network in precision medicine. Tomography. 2016;2(4):239-41.

52. Norton K-A, et al. Multiscale Agent-Based and Hybrid Modeling of the Tumor Immune Microenvironment. Processes (Basel, Switzerland). 2019;7(1):37.

53. Bindea $G$, et al. Natural immunity to cancer in humans. Curr Opin Immunol. 2010;22(2):215-22

54. Bindea $\mathrm{G}$, et al. Spatiotemporal dynamics of intratumoral immune cells reveal the immune landscape in human cancer. Immunity. 2013;39(4):782-95.

55. Farkona S, Diamandis EP, Blasutig IM. Cancer immunotherapy: the beginning of the end of cancer? BMC Med. 2016;14:73

56. Fridman $\mathrm{WH}$, et al. The immune contexture in human tumours: impact on clinical outcome. Nat Rev Cancer. 2012;12(4):298-306.

57. Galon J, et al. The continuum of cancer immunosurveillance: prognostic, predictive, and mechanistic signatures. Immunity. 2013;39(1):11-26.

58. Galon J, et al. Type, density, and location of immune cells within human colorectal tumors predict clinical outcome. Science. 2006; 313(5795):1960-4.

59. Galon J, et al. Towards the introduction of the 'Immunoscore' in the classification of malignant tumours. J Pathol. 2014:232(2):199-209.

60. Galon J, et al. Cancer classification using the Immunoscore: a worldwide task force. J Transl Med. 2012;10:205.

61. Wolchok JD, et al. Nivolumab plus ipilimumab in advanced melanoma. $N$ Engl J Med. 2013;369(2):122-33.

62. Saltz J, et al. Towards generation, management, and exploration of combined Radiomics and Pathomics datasets for Cancer research. AMIA Jt Summits Transl Sci Proc. 2017:2017:85-94.

63. Saltz J, et al. Spatial organization and molecular correlation of tumorinfiltrating lymphocytes using deep learning on pathology images. Cell Rep. 2018;23(1):181.

64. Levenson RM, Borowsky AD, Angelo M. Immunohistochemistry and mass spectrometry for highly multiplexed cellular molecular imaging. Lab Investig. 2015:95(4):397-405.

65. Koelzer $\mathrm{VH}$, et al. Precision immunoprofiling by image analysis and artificial intelligence Virchows Arch. 2019:474(4):511-22.

66. Krueger R, et al. Combining Unsupervised and Supervised Learning for Hierarchical Phenotype Analysis in Multi-Channel Image Data. Facetto: IEEE Trans Vis Comput Graph; 2019

67. Saylor J, et al. Spatial mapping of myeloid cells and macrophages by multiplexed tissue staining. Front Immunol. 2018;9:2925.

68. Roa-Pena $L$, et al. Keratin 17 identifies the most lethal molecular subtype of pancreatic cancer. Sci Rep. 2019;9(1):11239.

69. Babu S, et al. Keratin 17 is a sensitive and specific biomarker of urothelial neoplasia. Mod Pathol. 2019;32(5):717-24.

70. Escobar-Hoyos LF, et al. Keratin-17 promotes p27KIP1 nuclear export and degradation and offers potential prognostic utility. Cancer Res. 2015;75(17): 3650-62.

71. Escobar-Hoyos LF, et al. Keratin 17 in premalignant and malignant squamous lesions of the cervix: proteomic discovery and immunohistochemical validation as a diagnostic and prognostic biomarker. Mod Pathol. 2014;27(4):621-30

72. Zhang $\mathrm{W}$, et al. Fully automated 5-plex fluorescent immunohistochemistry with tyramide signal amplification and same species antibodies. Lab Investig. 2017:97(7):873-85.

73. Zhou W, et al. Image quality assessment: from error visibility to structural similarity. IEEE Trans Image Process. 2004;13(4):600-12.

74. Lambert JH. Photometria, sive, De mensura et gradibus luminis, colorum et umbrae; 1760 
75. Abousamra, S., et al., Weakly-Supervised Deep Stain Decomposition for Multiplex IHC Images, in IEEE International Symposium on Biomedical Imaging (ISBI), 2020. 2020.

76. Chen T, Chefd'hotel C. Deep Learning Based Automatic Immune Cell Detection for Immunohistochemistry Images. In: Machine Learning in Medical Imaging. Cham: Springer International Publishing; 2014.

77. Chen T, Srinivas C. Group sparsity model for stain unmixing in brightfield multiplex immunohistochemistry images. Comput Med Imaging Graph. 2015;46(Pt 1):30-9.

78. Duggal $R$, et al. SD-layer: stain Deconvolutional layer for CNNs in medical microscopic imaging. Cham: Springer International Publishing; 2017.

79. Macenko M, et al. A method for normalizing histology slides for quantitative analysis. In: Proceedings of the Sixth IEEE international conference on Symposium on Biomedical Imaging: From Nano to Macro. Boston: IEEE press; 2009. p. 1107-10.

80. Vahadane A, et al. Structure-preserving color normalization and sparse stain separation for histological images. IEEE Trans Med Imaging. 2016;35(8):1962-71.

81. Ronneberger O, Fischer P, Brox T. U-Net: Convolutional Networks for Biomedical Image Segmentation; 2015. p. 234-41.

\section{Publisher's Note}

Springer Nature remains neutral with regard to jurisdictional claims in published maps and institutional affiliations.

Ready to submit your research? Choose BMC and benefit from:

- fast, convenient online submission

- thorough peer review by experienced researchers in your field

- rapid publication on acceptance

- support for research data, including large and complex data types

- gold Open Access which fosters wider collaboration and increased citations

- maximum visibility for your research: over $100 \mathrm{M}$ website views per year

At BMC, research is always in progress.

Learn more biomedcentral.com/submissions 